\title{
High-Field Pulsed ENDOR with Intra-cavity Radiofrequency Coil
}

\section{G. Annino ${ }^{1}$ (D) H. Moons ${ }^{2} \cdot$ M. Fittipaldi ${ }^{3} \cdot$ S. Van Doorslaer ${ }^{4} \cdot$ E. Goovaerts $^{2}$}

Received: 13 July 2020 / Revised: 11 September 2020 / Accepted: 15 September 2020 /

Published online: 12 October 2020

(c) The Author(s) 2020

\begin{abstract}
This study compares the performance of two coil configurations for W-band pulsed ENDOR using a setup with both a radiofrequency 'hairpin' coil internal to a microwave non-radiative resonator and Helmholtz-like coils external to the resonator. Evaluation of the different coil performances is achieved via the ENDOR study of two model systems. The efficiencies of the coil configurations are first investigated numerically, showing that a higher radiofrequency current-to-magnetic field conversion factor can be achieved with the intra-cavity coil, with a similar radiofrequency magnetic field uniformity. This result is then confirmed by the broadband ENDOR spectra acquired with the two coil arrangements. A gain in the signal-to-noise ratio enabled by the internal coil of about a factor 10 was observed. In some cases, the high conversion factor of the intra-cavity coil led to a saturation of the ENDOR transitions. The possibility to implement a similar intra-cavity radiofrequency coil configuration in higher field spectrometers is finally discussed.
\end{abstract}

Electronic supplementary material The online version of this article (https://doi.org/10.1007/s0072 3-020-01269-z) contains supplementary material, which is available to authorized users.

G. Annino

geannino@ipcf.cnr.it

1 Istituto per i Processi Chimico-Fisici, IPCF-CNR, via G. Moruzzi 1, 56124 Pisa, Italy

2 ECM Laboratory, Department of Physics, University of Antwerp, Universiteitsplein 1, 2610 Antwerp, Belgium

3 Department of Physics and Astronomy and INSTM Research Unit, University of Florence, via G. Sansone 1, 50019 Sesto Fiorentino, Italy

4 BIMEF Laboratory, Department of Chemistry, University of Antwerp, Universiteitsplein 1, 2610 Antwerp, Belgium 


\section{Introduction}

Among the various techniques developed in the field of Electron Paramagnetic Resonances (EPR), the Electron-Nuclear Double Resonance (ENDOR) spectroscopy owes its popularity to the ability to provide detailed information on hyperfine interactions and on the electronic structure of paramagnetic centers. Initially proposed in 1956 by Feher as a transient technique [1-3], it mainly established itself in its continuous-wave (cw) version during the first decades [4, 5]. Pulsed ENDOR techniques, made appealing by potentially stronger as well as less distorted signals [6], became important several years after their initial proposal [7, 8], thanks to the development of the necessary electronics.

Nowadays, cw and pulsed ENDOR are both largely utilized in EPR laboratories. $\mathrm{cW}$ ENDOR requires the right balance between the longitudinal relaxation time of the electron spins and that of the nuclear spins. Usually, it provides a detectable signal only in a limited temperature range. On the other hand, pulsed ENDOR needs a relatively long electron spin relaxation time, which in general requires low temperatures [9].

For pulsed ENDOR, the main trend that emerged in the last decades has probably been the implementation at magnetic fields $\mathrm{B}_{0}$ higher than the standard $0.3 \mathrm{~T}$, supported by the higher sensitivity and spectral resolution expected at increasing $\mathrm{B}_{0}[10,11]$. Spectrometers operating at high magnetic fields, such as $3.3 \mathrm{~T}$ $(94 \mathrm{GHz})$ and $9.4 \mathrm{~T}(263 \mathrm{GHz})$, are now commercially available, equipped with ENDOR capabilities.

In $\mathrm{cw}$ ENDOR, the maximum signal is usually obtained when the nuclear spin transition is at least partially saturated [12]. An efficient cw ENDOR setup should, thus, be characterized by a high radiofrequency (rf) current-to-magnetic field conversion factor at the sample region [13], defined as the field $\mathrm{B}_{\mathrm{rf}}$ generated by the unitary current. In pulsed ENDOR, the rf irradiation must ideally invert the population of the nuclear spin levels [7, 8]. Here, an efficient rf irradiation is still more crucial to minimize the overall length of the pulse sequence and, therefore, maximize the revealed signal, thanks to the reduced spin relaxation effects. Moreover, the possibility to generate very short rf pulses can enable more advanced coherence transfer ENDOR experiments [14].

The highest $\mathrm{B}_{\mathrm{rf}}$ conversion factor can be obtained by surrounding the sample with the rf coil [5], because the rf magnetic fields are maximum close to the conductors where the rf currents flow. Such a solution is made problematic by the concurrent presence of a microwave (mw) resonator, usually given by a closed metallic cavity, which is essential to reach a good efficiency in the excitation and detection of the EPR transitions. The insertion of metallic components inside the mw cavity can lead to a distortion of its electromagnetic fields and a deterioration of its quality factor $Q$. Analogously, the metallic cavity can affect the $B_{\text {rf }}$ field of the coil. At high fields, these problems are exacerbated by the millimeter or submillimeter size of the mw cavity. Ideally, the rf coil should be made transparent to the mw radiation stored in the cavity or vice versa. 
Possible solutions combining an rf coil with a mw cavity were investigated since the birth of ENDOR spectroscopy. In 1956, Feher proposed a probe head with the mw cavity having conducting walls electrically thick with respect to the mw fields and electrically thin with respect to the rf fields of the surrounding if coil [3]. In 1958, Motchane et al. employed a setup based on a mw cavity with internal rf coil [15]. A sort of intermediate configuration was proposed in 1973 by Gruber et al., on the basis of a previous idea of Hyde [16, 17]: in their approach, the cylindrical wall of the cavity was cut in the form of a helix and employed itself as an rf coil. As in the case of the Motchane paper, the successful combination of the rf structure with the mw structure proposed by Gruber work was made possible by the peculiar field and current distribution of the $\mathrm{TE}_{011}$ mode of the cylindrical mw cavity.

The configurations with mw cavity housing an internal rf coil or having cylindrical wall acting as rf coil are the most commonly employed at standard frequencies [18]. At high fields, the necessary miniaturization of the probe head favored the solution with rf coil external to the cavity, implemented with the expedient of cutting several slits in the cylindrical body of the resonator [19-24], as proposed by Burghaus et al. to facilitate the penetration of the rf field [25]. However, from the point of view of the $B_{\text {rf }}$ conversion factor, the configuration with the $\mathrm{rf}$ coil external to the mw cavity is doubly inefficient in comparison to the one with the intra-cavity coil. First, because the sample is placed at a relatively large distance from the conductor forming the coil, then because the mw cavity partially shields the $B_{\text {rf }}$ field. The development of high-field ENDOR setups based on intra-cavity rf coils has probably been hindered by the belief that such a solution is unfeasible due to the small mw cavity size, the possible microphonic noise, and the reduced mw $Q$ factor.

The aim of the work here presented was to explore the possibility of high-field pulsed ENDOR spectroscopy with an intra-cavity rf coil and to assess the obtained sensitivity with respect to the case of external coils. The employed configuration is based on a non-radiative (nr) mw resonator [26], equipped with internal 'hairpin' coil, following an approach similar to that demonstrated in high-field Dynamic Nuclear Polarization [27, 28], as part of a work aiming at the combination of EPR and NMR techniques [29]. A similar concept was employed to introduce a low-frequency electric field in a mw cavity [30].

Preliminary W-band ENDOR results obtained with a sample of protonated 1,3-bis(diphenylene)-2-phenyl-allyl (BDPA) radical dissolved in polystyrene were presented in [31]. Here, a more systematic study, mainly conducted on a single crystal of $\gamma$-irradiated LiF sample, is presented. Particular attention is dedicated to the broadband capabilities of the proposed solution and on its advantages in terms of sensitivity. Determining the exact contribution of a single component to the overall sensitivity of a spectrometer, in our case the intra-cavity hairpin coil, is a delicate task, in which all the factors concurring to the response of a typical ENDOR spectrometer have to be considered. To give a definitive answer to this question, the employed probe head was equipped with both external coils in the typical Helmholtz-like configuration and with the intra-cavity hairpin coil. Thus, the results 
obtained with the hairpin coil were assessed by comparison with the results of the external coils, acquired in identical conditions.

The structure of the paper is the following. Section 2 illustrates the structure of the 'double-coil' probe head employed in this work. The distribution and the intensity of the $\mathrm{B}_{\mathrm{rf}}$ field generated by the coils are investigated numerically in Sect. 3 . Section 4 reports the ENDOR results obtained with the LiF and BDPA in polystyrene samples. The obtained results are discussed in Sect. 5, together with the perspectives of higher frequency applications. Finally, Sect. 6 reports the main conclusions.

\section{Experimental Setup}

The W-band probe head employed in this work is based on a $\mathrm{nr}$ resonator similar to the one described in [26]. In general, the main characteristic of the $\mathrm{nr}$ resonator is the combination of a single-mode spectrum with an open configuration [32], which can be obtained with a simple modular structure [33, 34]. The ease of construction and setup, the absence of mode degeneracy, and the state-of-the-art performances demonstrated in high-field EPR applications [26, 27] make the nr cavities an ideal candidate for the exploration of new experimental approaches.

The main details of the probe head are illustrated in Fig. 1 (top), showing a sector of the complete structure. The gray-shaded regions represent the empty parts of the probe head, whose core is composed of a cylindrical cavity with radius $r=1.9 \mathrm{~mm}$ and variable height $h$. The gap $g$ between the two parts of the cavity was fixed to $1.45 \mathrm{~mm}$. Coaxial holes with diameter $d=1.2 \mathrm{~mm}$ were drilled in the plungers of the cavity, which are movable to enable the tuning of the resonance mode. Grooves were cut in the two metallic plates composing the nr resonator, to accommodate the external rf coils (blue-shaded regions of Fig. 1). The latter is given by loops with a $6 \mathrm{~mm}$ diameter, each composed by 3 windings of insulated copper wire of $250 \mu \mathrm{m}$ diameter. The two loops are placed at a distance of about $5 \mathrm{~mm}$ from each other. The U-shaped hairpin coil, represented by the green-shaded region of Fig. 1, is obtained by gluing an insulated copper wire of $80 \mu \mathrm{m}$ diameter along a standard quartz sample holder $(0.60 \mathrm{~mm}$ i.d. and $0.84 \mathrm{~mm}$ o.d.). The inset in the upper part of Fig. 1 shows the basic version of the nr resonator, composed by a cylindrical cavity, a relatively large gap, and the holes in the plungers, together with the typical distribution of the mw electric field of the $\mathrm{TE}_{011}$ mode.

The lower part of Fig. 1 shows on the left a picture of one of the two plates forming the $\mathrm{nr}$ resonator, together with the external coils, and on the right a picture of the sample holder equipped with the hairpin coil. The insertion in the nr resonator of the thin conducting wires forming the hairpin coil does not introduce appreciable effects on the mw quality factor of its $\mathrm{TE}_{011}$ mode, the one of interest for our measurements, thanks to the orthogonality of the wires with respect to the mw electric field lines. For the resonator of Fig. 1, the unloaded quality factor was measured to be $Q_{0}=2800$ at $94 \mathrm{GHz}$, independently of the presence of the hairpin coil within the measurement uncertainty $( \pm 10 \%)$ allowed by the employed mw bridge. 

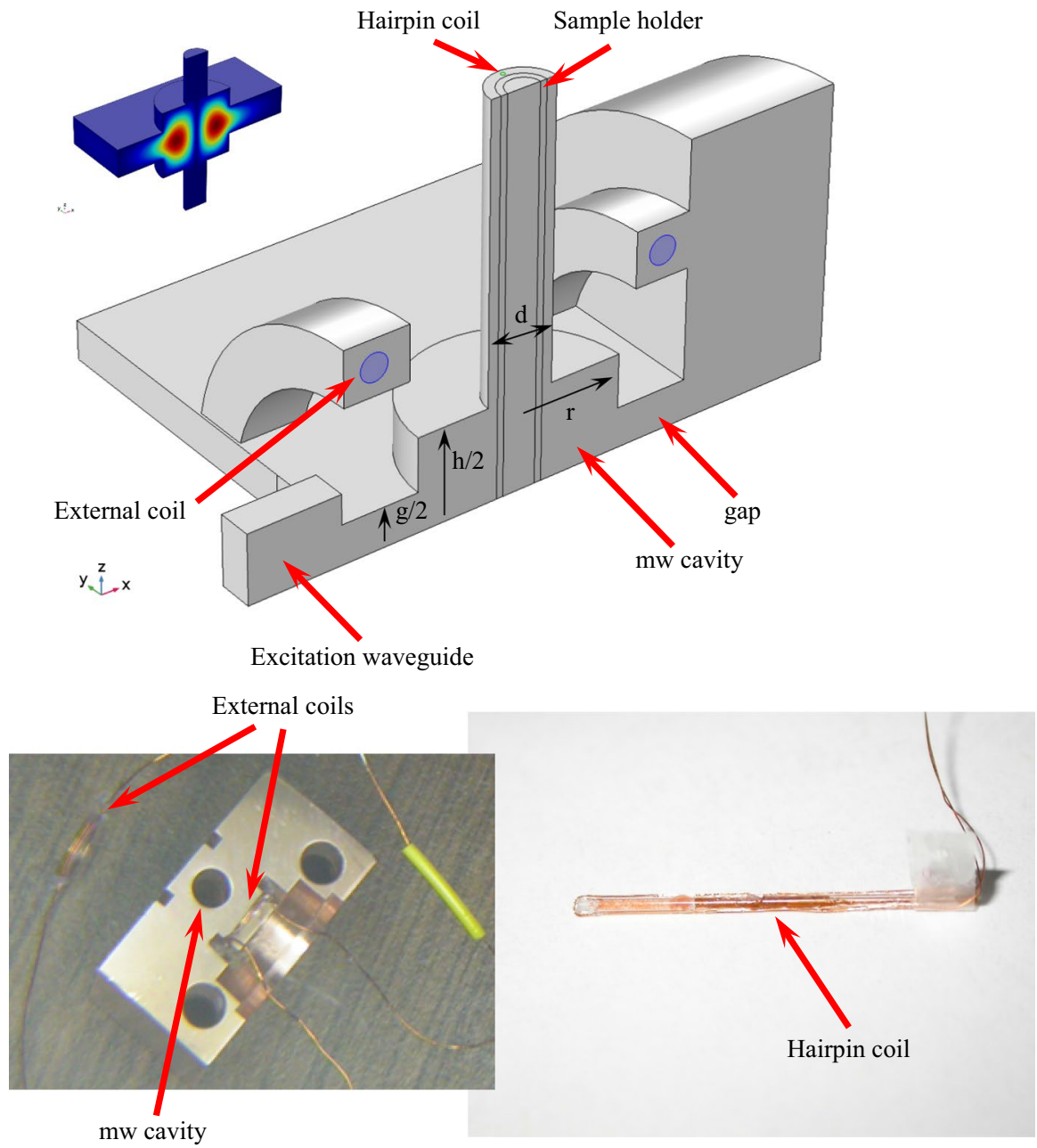

Fig. 1 Details of the probe head. Upper part: structure employed in the modeling, representing a onequarter sector of the whole structure, which can be obtained by mirror symmetry with respect to the $x y$ and $x z$ planes. The main components and dimensions are indicated. Inset in the upper part: basic version of the $\mathrm{nr}$ resonator, together with the typical distribution of the mw electric field of the $\mathrm{TE}_{011}$ mode. In this case, the whole structure can be obtained by mirror symmetry with respect to the $x z$ plane. Lower part: on the left, one of the plates forming the nr resonator, together with the external coils; on the right, sample holder equipped with the hairpin coil (color figure online)

\section{Simulations of the rf Fields}

The efficiency of the internal and external coils was first investigated, both in terms of $B_{\text {rf }}$ field distribution and current-to-field conversion factor, by means of numerical modeling based on the software Multiphysics (Comsol Inc., Sweden). The 
calculations were conducted on the structure of Fig. 1, corresponding to a quarter of the entire structure, assuming an rf current of $1 \mathrm{~A}$ at a frequency of $135 \mathrm{MHz}$. The field in the entire structure can be obtained from that in the modeled volume by symmetry.

Figure 2 shows the main results of the modeling. In particular, the upper part of Fig. 2 shows the norm of the $\mathrm{B}_{\mathrm{rf}}$ conversion factor on the surface of the structure for the hairpin coil (on the left) and for the Helmholtz-like coils (on the right). In the case of the hairpin coil, the conversion factor is quite intense along the axis of the resonator, in the region internal to the cavity. Outside the cavity, the $\left|B_{\mathrm{rf}}\right|$ conversion factor is mainly concentrated between the wires of the coil and the close metallic surface, due to the strong induced currents. For the Helmholtz coils, the $\left|B_{\mathrm{rf}}\right|$ conversion factor is very intense around the wires, more than for the hairpin coil owing to the higher effective current due to the 3 windings, but weaker inside the resonator.

A quantitative comparison is made in the lower part of Fig. 2, showing the $\left|\mathrm{B}_{\mathrm{rf}}\right|$ conversion factor along the axis of the resonator. In the reported graphs, the origin of the abscissa axis corresponds to the center of the resonator.
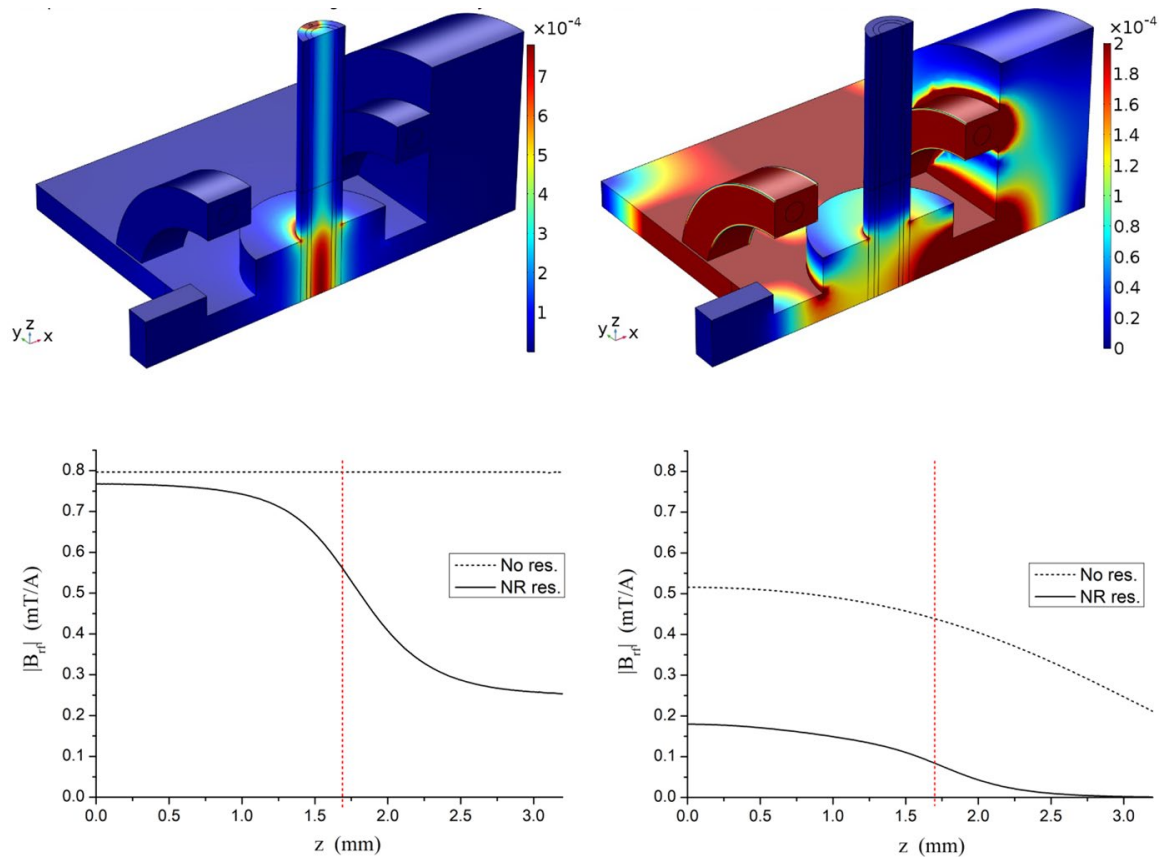

Fig. 2 Results of numerical simulations on the $\left|B_{\mathrm{rf}}\right|$ field generated by the coils, assuming an rf current of 1 A. Upper part: on the left, the $\left|B_{\mathrm{rf}}\right|$ field generated by the intra-cavity coil; on the right, the field generated by the external coils. The color scales are different. Lower part: on the left, the $\left|B_{\text {rf }}\right|$ field generated by the hairpin coil along the $z$ axis, both in the case of the coil inside the nr resonator (solid line) and the coil without the nr resonator (dashed line); on the right, the field generated by the external coils along the $z$ axis, both in the case of the coil external to the $\mathrm{nr}$ resonator (solid line) and the coil without the $\mathrm{nr}$ resonator (dashed line). The red-dashed line represents the border of the mw cavity (color figure online) 
The bottom-left part of Fig. 2 shows the fields calculated for the hairpin coil with the $\mathrm{nr}$ resonator (solid line) and without the nr resonator (dashed line). The conversion factor in the center of the resonator is $0.77 \mathrm{mT} / \mathrm{A}$ for the hairpin with the $\mathrm{nr}$ resonator and $0.8 \mathrm{mT} / \mathrm{A}$ in the case of the hairpin without the $\mathrm{nr}$ resonator. On the border of the nr cavity, indicated in Fig. 2 by a red-dashed line, the conversion factor decreases up to $0.56 \mathrm{mT} / \mathrm{A}$. In the case of the external coils (bottom-right part of Fig. 2), the conversion factor in the center of the resonator is $0.18 \mathrm{mT} / \mathrm{A}$ for the coils with the $\mathrm{nr}$ resonator and $0.52 \mathrm{mT} / \mathrm{A}$ for the coils without the resonator. On the border of the nr cavity, the conversion factor is $0.084 \mathrm{mT} / \mathrm{A}$.

Accordingly, the magnetic field $\left|B_{\text {rfi }}\right|$ generated by the hairpin coil in the portion of sample holder placed inside the cavity is about 4 times stronger than the magnetic field $\left|B_{\text {rfe }}\right|$ generated by the external coils, for the same rf current. The uniformity of $\left|B_{\mathrm{rfi}}\right|$ is better than that of $\left|B_{\mathrm{rfe}}\right|$ along the axis of the sample holder ( $z$ axis of Fig. 1$)$, and worse in the orthogonal (xy) plane. The overall uniformity is similar, considering the data shown in Fig. S1 of the Supplementary Material (SM) available with the online version of this article.

The conversion factor of the hairpin coil in the center of the resonator, $\left|B_{\mathrm{rf}}\right|=0.77$ $\mathrm{mT} / \mathrm{A}$, is comparable with the value calculated for the 4-wire coil internal to the $35 \mathrm{GHz}$ resonator investigated in [35], as well as with the value calculated for the Bruker ENDOR resonator operating at the same frequency [35]. For the Helmholtzlike coils, the obtained value $\left|\mathrm{B}_{\mathrm{rf}}\right|=0.18 \mathrm{mT} / \mathrm{A}$ is comparable with the conversion factor of the coils external to the slotted cavity operating at $263 \mathrm{GHz}$ in the Bruker spectrometer presented in [24].

\section{Pulsed ENDOR Analysis}

The W-band ENDOR measurements were performed with a Bruker Elexsys E680 spectrometer, equipped with a mw source delivering $4.4 \mathrm{~mW}$. In general, such a relatively low power is not ideal for pulsed ENDOR measurements. The spectrometer is equipped with a Bruker rf amplifier model B-LTX300 delivering a maximum power of $300 \mathrm{~W}$. The rf coils were inserted in a non-resonant $\mathrm{rf}$ circuit fed by the rf source and terminated by a $50 \Omega$ load. The power delivered to the load was monitored measuring the voltage at its ends. Both the external and the internal coils were present during the measurements, to acquire the spectra in the same conditions. Switching from one rf configuration to the other required just a change in the rf connections.

The measurements, all conducted at room temperature $T$, concerned two samples: a single crystal of $\gamma$-irradiated LiF with dimensions of the order of $0.3 \times 0.3 \times 1 \mathrm{~mm}^{3}$, and a powder of $1 \%$ BDPA radical dissolved in polystyrene.

\subsection{ENDOR Analysis of $\boldsymbol{\gamma}$-Irradiated LiF}

$\gamma$-Irradiated LiF is an ideal test sample for a broadband ENDOR spectrometer, thanks to its well-investigated and rich ENDOR spectrum, originating from the 

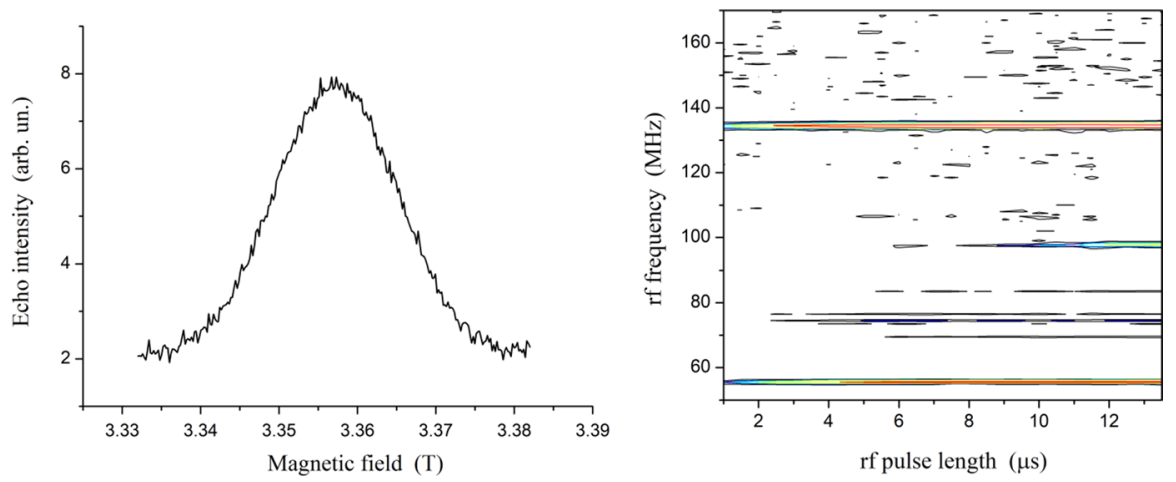

Fig. 3 Left: Typical ESE signal obtained with the LiF sample. Pulse parameters: $\pi / 2=348$ ns, $\tau=1200 \mathrm{~ns}$. Shot repetition time $2.5 \mathrm{~ms}$, shots/point 100. 2 scans. Right: Level plot of Mims ENDOR of the LiF sample with the intra-cavity coil, acquired at variable rf frequency and pulse length. Pulse parameters: $\pi / 2=348 \mathrm{~ns}, \tau=600 \mathrm{~ns}$. Shot repetition time $2.14 \mathrm{~ms}$, shots/point $250.1 \mathrm{scan}$

strong hyperfine interactions of the unpaired electron with the nuclei in many inequivalent sites [36-39]. The typical field-swept electron-spin-echo (ESE) spectrum obtained from the LiF sample is shown in the left part of Fig. 3. The right part of Fig. 3 shows the ENDOR signal obtained by means of the hairpin coil with a Mims pulse sequence [7], in a 2D scan over the rf frequency and the rf pulse length $T_{\text {rf }}$. The ${ }^{19} \mathrm{~F}$ line at $135 \mathrm{MHz}$ and the ${ }^{7} \mathrm{Li}$ line at $55 \mathrm{MHz}$ are clearly visible [21], together with a line at $96 \mathrm{MHz}$ and a multiplet centered around $77 \mathrm{MHz}$.

The details of the ${ }^{19} \mathrm{~F}$ Mims signal at $135 \mathrm{MHz}$, acquired with the hairpin coil, are shown in the left part of Fig. 4. The analogous signal, acquired with the external coils, is shown in the right part of Fig. 4.

A direct comparison between the signal-to-noise ratios $(\mathrm{S} / \mathrm{N})$ of the spectra shown in Fig. 4 is not possible owing to a rf saturation of the ENDOR signal, close to the Larmor frequency, obtained with the intra-cavity coil. This effect is
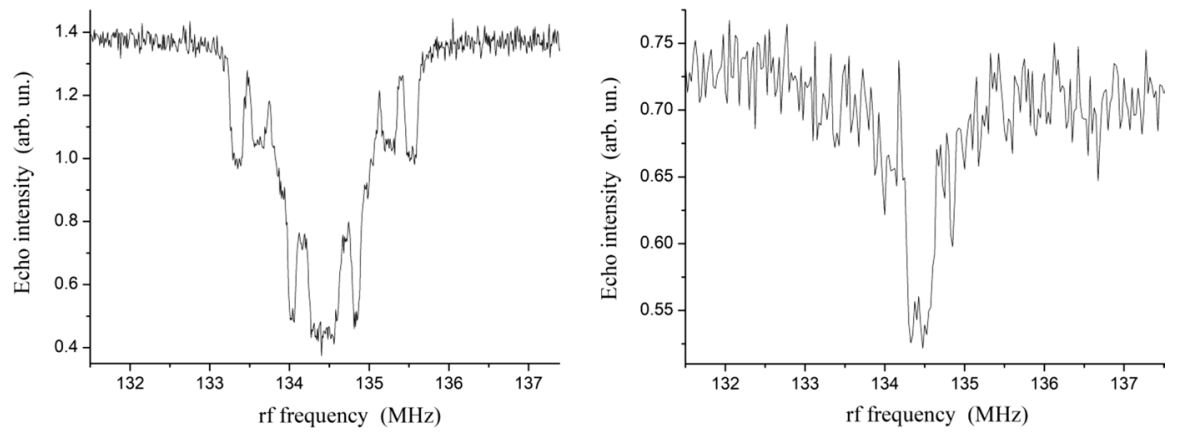

Fig. 4 Mims ENDOR on the ${ }^{19} \mathrm{~F}$ line at $135 \mathrm{MHz}$. Left: Intra-cavity coil (1 scan). Right: External coils ( 2 scans). Pulse parameters: $\pi / 2=348 \mathrm{~ns}, \tau=700 \mathrm{~ns}, T_{\mathrm{rf}}=12 \mu \mathrm{s}$. Shot repetition time $2.5 \mathrm{~ms}$, shots/point 250 
shown in Fig. 5, reporting the ESE signal obtained setting the rf to the center of the ${ }^{19} \mathrm{~F}$ ENDOR line of Fig. 4 and varying the rf power. The obtained signal is completely saturated at the maximum available rf power, being flat up to an attenuation of this power by about $3 \mathrm{~dB}$.

The inset in the left part of Fig. 5 shows the ${ }^{19} \mathrm{~F}$ ENDOR line at full rf power, whereas the inset in the right part shows the same line at rf power attenuated by $3 \mathrm{~dB}$. A comparison between these two spectra shows that the peak at $133.3 \mathrm{MHz}$ is not saturated and displays a shape very close to the one obtained with the external coils (Fig. 4, Right). Thus, it can be employed to determine the S/N obtained with the two kind of coils.

Figure 6 reports the comparison of part of the ${ }^{19} \mathrm{~F}$ Mims ENDOR spectra obtained with the internal and the external coils. In the former case, the signal was obtained with a single scan, in the latter with 50 scans. According to these curves, in a single scan the $\mathrm{S} / \mathrm{N}$ of the ${ }^{19} \mathrm{~F}$ line obtained with the hairpin coil is higher by a factor 14 with respect to the case of the external coils. Moreover, a check of the voltage on the $50 \Omega$ load during the acquisition of the ${ }^{19} \mathrm{~F}$ lines indicated an $\mathrm{rf}$ current on the hairpin coil lower than that in the external coils.

The ${ }^{7} \mathrm{Li}$ Mims ENDOR signal at $55 \mathrm{MHz}$ is reported in Fig. 7 (top), in the left part as acquired with the hairpin coil and in the right part as acquired with the external coils. As in the case of the ${ }^{19} \mathrm{~F}$ Mims ENDOR line at $135 \mathrm{MHz}$, the central peak

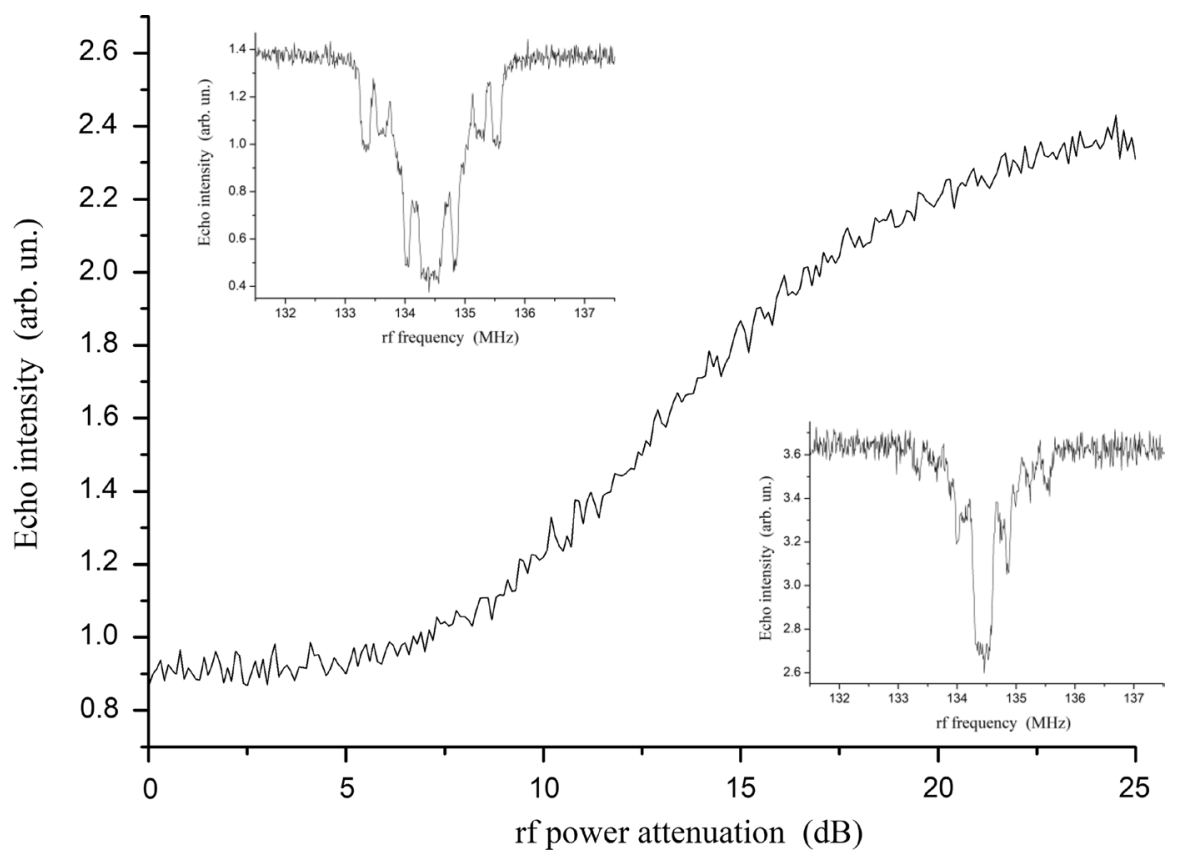

Fig.5 Mims ENDOR signal at a fixed rf frequency of $134.4 \mathrm{MHz}$ and variable rf power attenuation, obtained with the intra-cavity coil ( 2 scans). Pulse parameters: $\pi / 2=348 \mathrm{~ns}, \tau=700 \mathrm{~ns}, T_{\mathrm{rf}}=12 \mu \mathrm{s}$. Shot repetition time $2.5 \mathrm{~ms}$, shots/point 250. Inset to the left: Mims ENDOR on the ${ }^{19} \mathrm{~F}$ line, full $\mathrm{rf}$ power. Inset to the right: Mims ENDOR on the ${ }^{19} \mathrm{~F}$ line, rf power attenuated by $3 \mathrm{~dB}$. Pulse parameters as for the main figure 


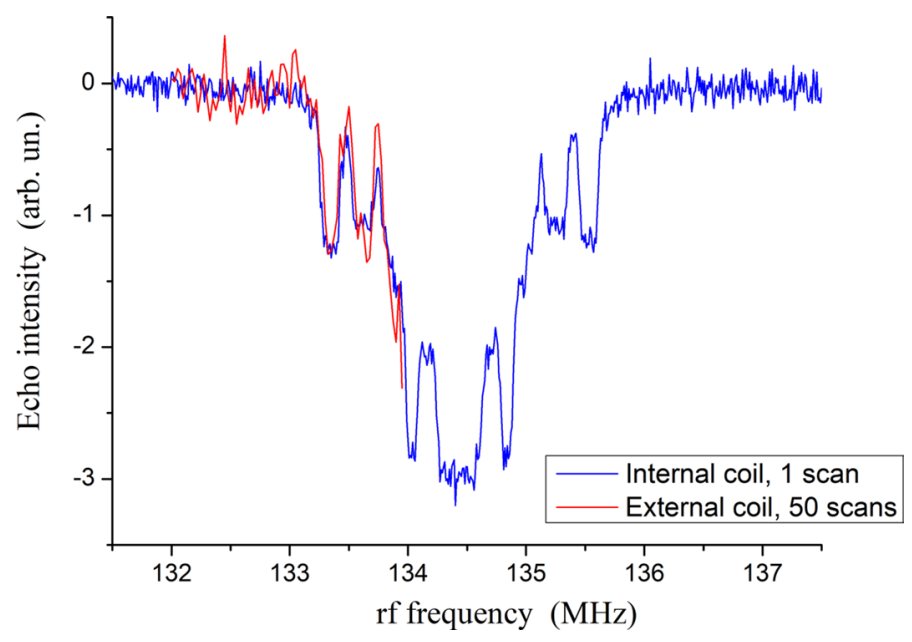

Fig. 6 Mims ENDOR on the ${ }^{19} \mathrm{~F}$ line at $135 \mathrm{MHz}$. Blue line: Intra-cavity coil, 1 scan. Red line: External coils, 50 scans. For both curves, the measurement parameters are $\pi / 2=348 \mathrm{~ns}, \tau=700 \mathrm{~ns}, T_{\mathrm{rf}}=12 \mu \mathrm{s}$. Shot repetition time $2.5 \mathrm{~ms}$, shots/point 250 (color figure online)

at $55.5 \mathrm{MHz}$ is saturated, as shown by the inset in the top-left part of Fig. 7, reporting the signal at $55.5 \mathrm{MHz}$ as a function of the rf power.

The Mims ENDOR signal at $97 \mathrm{MHz}$ is shown in detail in the lower part of Fig. 7, reporting in the left part the spectrum obtained with the hairpin coil and in the right part that obtained with the external coil. It is attributed to the hyperfine interaction of the paramagnetic center with the nearest-neighbor fluorines, as discussed in [35]. In cw ENDOR, this signal is reported to be very broad. This is consistent with the absence of rf power saturation observed in our measurements, as shown by the inset in the bottom-left part of Fig. 7, reporting the ENDOR signal of the second peak $(97.74 \mathrm{MHz})$ as a function of the rf power.

The optimal length $T_{\text {rf }}$ of the rf $\pi$ pulse was estimated on this peak and resulted about $T_{\mathrm{rf}}=15 \mu \mathrm{s}$. The related nutation curve is reported in Fig. S2 of the Supplementary Material (SM) available with the online version of this article. The corresponding quantity for the external coils would exceed the maximum interval between the second and the third pulse of the Mims sequence allowed by the ESE signal, which is about $45 \mu \mathrm{s}$.

To demonstrate the broad-band capability of the hairpin configuration, the Mims ENDOR spectrum of the LiF sample was acquired in the interval 10-90 MHz. It is shown in the upper part of Fig. 8. As expected, it is quite rich in resonance lines. More resolved spectra are reported in the lower part of Fig. 8 for the strong lines at $18.5 \mathrm{MHz}$ and $45 \mathrm{MHz}$, which are attributed to overtone resonances with the ${ }^{7} \mathrm{Li}$ signal at $55.5 \mathrm{MHz}$ and with the ${ }^{19} \mathrm{~F}$ signal at $135 \mathrm{MHz}$, respectively.

The effect on the 10-90 MHz Mims spectrum of a $20^{\circ}$ rotation of the $\mathrm{B}_{0}$ with respect to the sample is reported in Fig. S3 of the SM. Moreover, this figure 

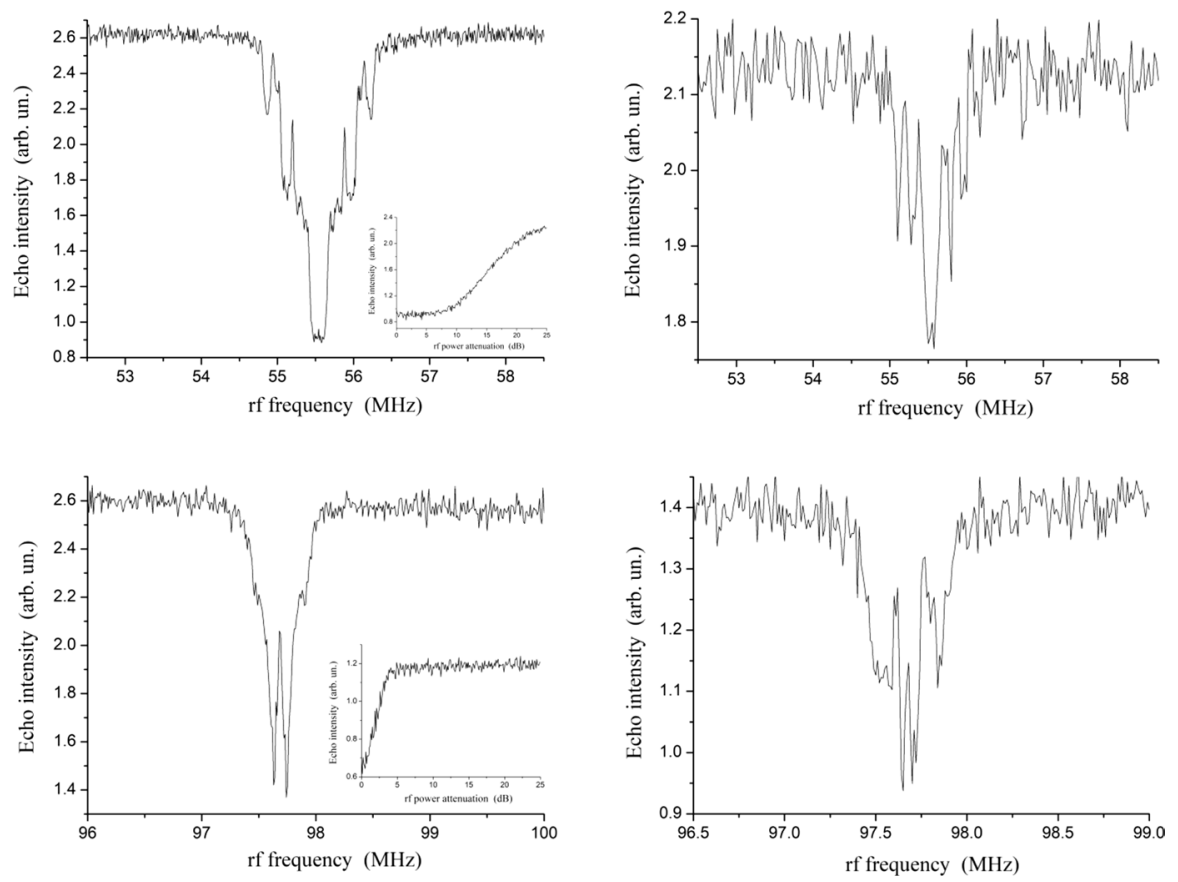

Fig. 7 Top: Mims ENDOR on the ${ }^{7} \mathrm{Li}$ line at $55 \mathrm{MHz}$. Left: Intra-cavity coil (2 scans). Right: External coils (1 scan). Inset: Mims ENDOR signal at a fixed rf frequency of $55.55 \mathrm{MHz}$ and variable rf power attenuation, obtained with the intra-cavity coil (2 scans). Bottom: Mims ENDOR on the line at 97.7 MHz. Left: Intra-cavity coil (2 scans). Right: External coils (3 scans). Inset: Mims ENDOR signal at a fixed rf frequency of $97.74 \mathrm{MHz}$ and variable rf power attenuation, obtained with the intra-cavity coil ( 1 scan). For all curves, the measurement parameters are $\pi / 2=348 \mathrm{~ns}, \tau=700 \mathrm{~ns}, T_{\mathrm{rf}}=12 \mu \mathrm{s}$. Shot repetition time $2.5 \mathrm{~ms}$, shots/point 250

shows some of the sharpest peaks that were observed, useful for defining the spectral resolution obtained with the intra-cavity coil.

The performance of the set-up for ENDOR measurement was also investigated using the Davies pulse sequence [8]. The spectra obtained with the hairpin coil are reported in Fig. S4 of the SM.

\subsection{ENDOR Analysis of BDPA Radical in Polystyrene}

Protonated BDPA dissolved in polystyrene was chosen as a model sample for those cases in which the sample is amorphous and fills completely the sample holder. The ENDOR experiments gave signals both on the real and the imaginary (out-of-phase) components of the ESE, for which the spectra are shown in the inset of Fig. 9. Figure 9 shows on the left the contour plot of the Mims ENDOR signal obtained in a $2 \mathrm{D}$ scan versus the rf frequency and the rf pulse length, obtained by means of the hairpin coil from the real part of the ESE. This clearly shows the expected ${ }^{1} \mathrm{H}$ ENDOR signal around $142.8 \mathrm{MHz}$, which will be further discussed below. 

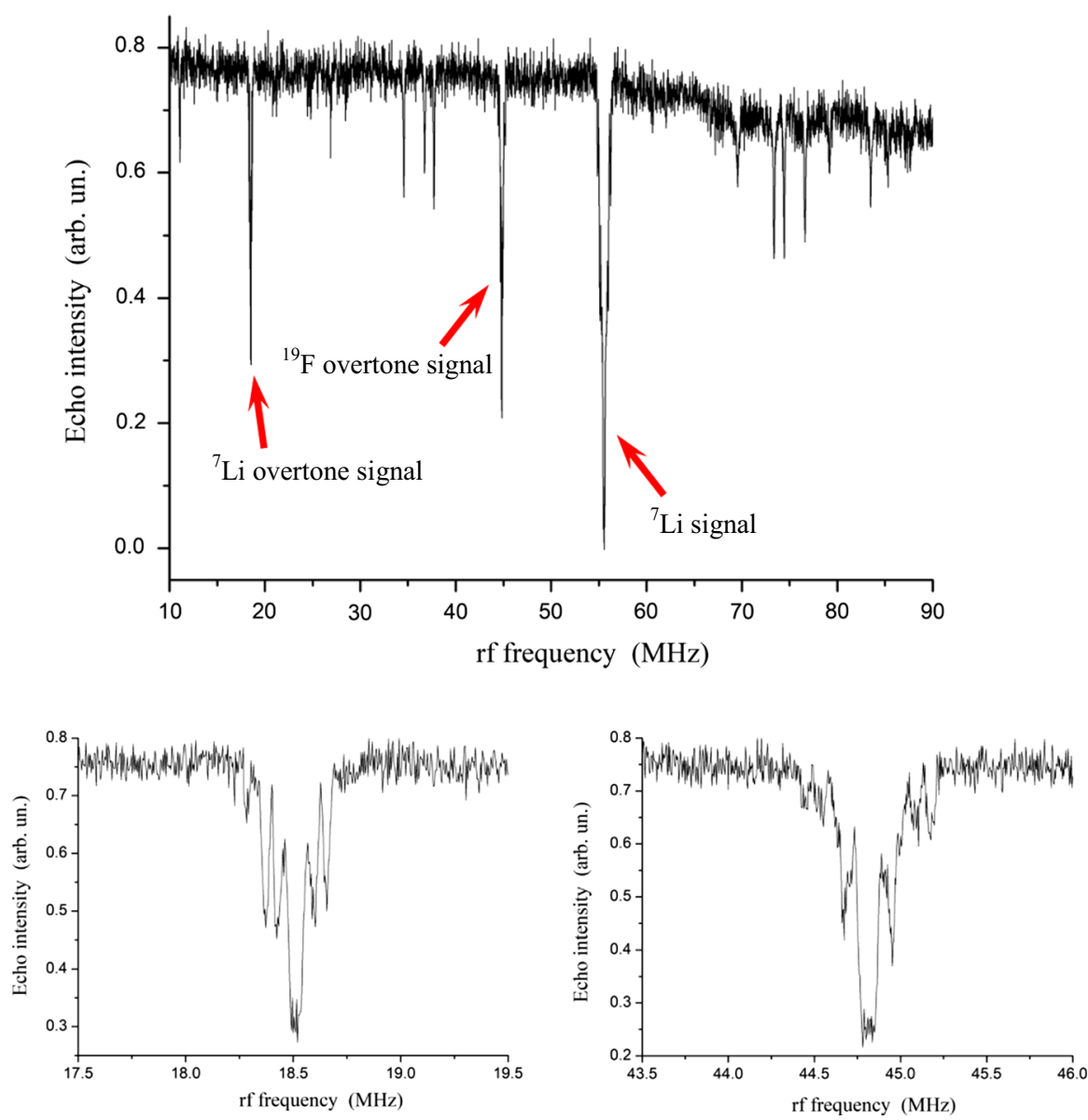

Fig. 8 Top: Mims ENDOR of the LiF sample on the frequency interval 10-90 MHz, obtained with the intra-cavity coil ( $1 \mathrm{scan}$ ). The ${ }^{7} \mathrm{Li}$ line and the overtone lines of the ${ }^{7} \mathrm{Li}$ and ${ }^{19} \mathrm{~F}$ are indicated. Acquisition mode: standard. Bottom-left: Mims ENDOR on the signal at $18.5 \mathrm{MHz}$ (1 scan). Bottom-right: Mims ENDOR on the signal $44.8 \mathrm{MHz}$ ( 1 scan). For all curves, the measurement parameters are $\pi / 2=348 \mathrm{~ns}$, $\tau=700 \mathrm{~ns}, T_{\mathrm{rf}}=12 \mu \mathrm{s}$. Shot repetition time $2.5 \mathrm{~ms}$, shots/point 250

The right part of the figure reports the corresponding contour plot from the outof-phase component of the ESE. Here, an intense signal is visible around $124 \mathrm{MHz}$, which is strongest on the out-of-phase component of the ESE. It is characterized by a much shorter modulation period in the length of the rf pulse, of the order of $4 \mu \mathrm{s}$. The same modulation effect appeared also in signals at frequencies in the 60-120 MHz range (Fig. S5 of the SM). These signals appear to originate in the sample holder. Their identification requires additional experiments and is beyond the scope of this paper.

The left part of Fig. 10 reports a Mims ENDOR sweep around the ${ }^{1} \mathrm{H}$ proton peak (10 scans), obtained with the hairpin coil. The right part of the figure reports the signal obtained in similar conditions by means of the external 

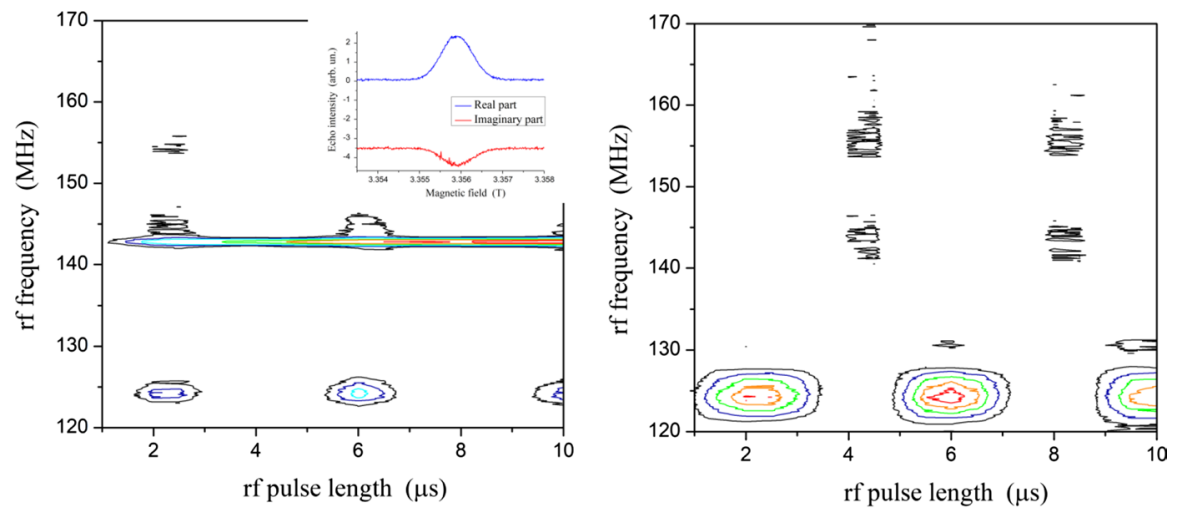

Fig. 9 Contour plots of Mims ENDOR of the BDPA in polystyrene sample with the intra-cavity coil, obtained with variable rf frequency and pulse length $(1 \mathrm{scan})$. Pulse parameters: $\pi / 2=348 \mathrm{~ns}, \tau=600 \mathrm{~ns}$. Shot repetition time $2.14 \mathrm{~ms}$, shots/point 250. rf power attenuated by $3 \mathrm{~dB}$. Left: Signal obtained from the real part of the ESE. Right: Signal obtained from the imaginary (out-of-phase) part of the ESE. Inset: Typical ESE signal obtained with the BDPA in polystyrene sample. Pulse parameters: $\pi / 2=348 \mathrm{~ns}$, $\tau=1200 \mathrm{~ns}$. Shot repetition time $1 \mathrm{~ms}$, shots/point 100

coil. The baseline of the signal obtained with the hairpin coil is distorted by the presence of weaker and broader signals, not visible with the external coils, as shown by Fig. S6 of the SM. On the other hand, the signal at $124 \mathrm{MHz}$ is visible, although very weakly, also with the external coil, again mainly on the outof-phase component of the ESE, with and without the internal coil. Fig. S7 of the SM shows the Mims and Davies signals around $124 \mathrm{MHz}$, obtained with the external coils.

In the case of the ${ }^{1} \mathrm{H}$ ENDOR signal of the BDPA dissolved in polystyrene, the $\mathrm{S} / \mathrm{N}$ obtained with the intra-cavity coil is about 8 times higher than that obtained with the external coils, in line with the higher sensitivity determined above for the ENDOR signals in irradiated LiF.
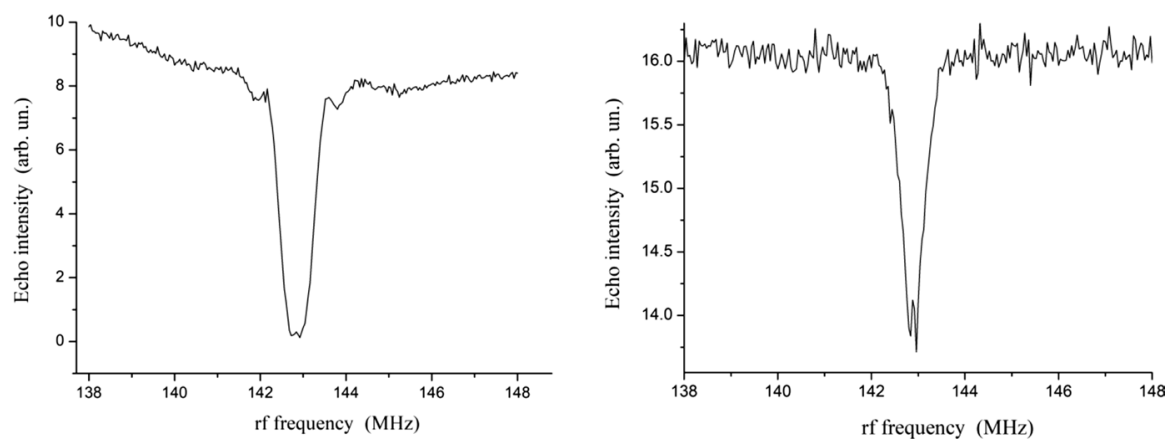

Fig. 10 Mims ENDOR on the ${ }^{1} \mathrm{H}$ line at $142.8 \mathrm{MHz}$ of the BDPA in polystyrene sample measured with the intra-cavity coil (Left) and the external coils (Right). Pulse parameters: $\pi / 2=348 \mathrm{~ns}, \tau=700 \mathrm{~ns}$, $T_{\mathrm{rf}}=10 \mu \mathrm{s}$. Shot repetition time $2.14 \mathrm{~ms}$, shots/point 250, 10 scans 


\section{Discussion and Perspectives}

According to the literature, the main drawbacks related to the use of intra-cavity rf coils in high-field ENDOR are the possible noise due to vibrations, the reduction of the quality factor of the mw cavity, and the difficulties of the necessary miniaturization $[14,25]$.

In the spectra acquired in this work, there was no clear evidence of noise induced by the rf irradiation, as confirmed by the presence of sharp peaks. Further specific checks were done for a better assessment of this possible issue. The noise floor was determined with and without the $\mathrm{rf}$ irradiation in a region of the LiF ENDOR spectrum around $126 \mathrm{MHz}$, in principle free from resonances. The resulting curves show no variation in the noise level, as reported in Fig. S8 of the SM. Analogously, the change of the shot repetition time in the acquisitions does not alter the shape of the ENDOR lines, as one could expect in case of rfinduced vibrations. This is true also for the anomalous signals observed in the 60-120 MHz frequency range of the Mims ENDOR of the BDPA radical, as shown in Fig. S9 of the SM. A further analysis is needed to determine the origin of these signals.

Possible effects deriving from the heating of the intra-cavity coil were checked acquiring the ENDOR both in standard and in stochastic mode [40]. In the case of the BDPA radical in polystyrene, no effects were observed, as shown in Fig. S10 of the SM. At very low T, the situation could be different due to the direct contact of the wires carrying the rf current with the sample holder. On the other hand, the efficiency of the intra-cavity coil proved much higher in comparison to the external coil, meaning that much less $\mathrm{rf}$ power is needed to obtain the same signal of the external coil. For instance, in the case of the ${ }^{19} \mathrm{~F}$ Mims line at $135 \mathrm{MHz}$, a comparison between Figs. 4 and 5 shows that the $\mathrm{S} / \mathrm{N}$ value of the external coil can be obtained employing about 2 orders of magnitude $(18 \mathrm{~dB})$ less rf power with the intra-cavity coil. Such a big difference is due to the fact that the efficiency of the rf coil in pulsed ENDOR is related to the rf current, which is proportional to the frequency of the nuclear spin nutation, whereas the heating effects are proportional to the square of the rf current. Consequently, the use of the intra-cavity coil could be advantageous also at very low $T$.

The insertion of the hairpin coil in the nr resonator proved to be not problematic from the point of view of the $Q$ factor of the resonator, consistently with the results reported in [27]. A direct confirmation that the intra-cavity coil does not affect the response of the ENDOR spectrometer is given in Fig. S11 of the SM, showing the Mims ENDOR signal of the ${ }^{1} \mathrm{H}$ peak of the BDPA radical dissolved in polystyrene obtained with the external coils before and after the gluing of the hairpin coil on the sample holder. Within the measurement uncertainty, the two $\mathrm{S} / \mathrm{N}$ ratios coincide.

In view of possible applications of the U-shaped intra-cavity coil to higher frequencies, one of the key aspects is the simplification that has been introduced with respect to the standard, 4-wire saddle coil [35], while keeping an rf field homogeneity similar to that of the external coils. In its easiest implementation, 
the insertion of the hairpin coil in the mw resonator just requires access holes slightly larger than the sample holder. In this respect, the use of $\mathrm{nr}$ resonators can be very helpful, thanks to their ability to keep a confined mw mode also in the limit case of axial holes having the same diameter of the cavity [32, 41]. In earlier work, an $\mathrm{nr}$ cavity resonating on the $\mathrm{TE}_{011}$ mode has been investigated up to about $360 \mathrm{GHz}$ (G. Annino, unpubl.). At $285 \mathrm{GHz}$, their quality factor at room T can be as high as $Q_{0}=3300$, as shown by Fig. S12 of the SM.

The key aspect behind the obtained results is the insertion in the resonator of thin conducting wires, in a manner that minimizes the distortion of the microwave field distribution. This can be obtained if the microwave electric field is mainly orthogonal to the surface of the wires and the magnetic field is mainly parallel to this surface. The ideal case is that of a cylindrical cavity operating in the $\mathrm{TE}_{011}$ mode, loaded by a cylindrical sample holder coaxial to the cavity. This configuration preserves the original cylindrical symmetry of the cavity, keeping a simple structure for the electromagnetic fields of the $\mathrm{TE}_{011}$ mode of this system, which is characterized by just a single component of the electric field (the azimuthal one) and two components of the magnetic field (the axial one and the radial one) [41]. As a consequence, it is possible and easy to respect almost everywhere the optimal boundary conditions between the fields and the surface of the wires forming the intra-cavity coil, so minimizing the distortion of the microwave mode and the degradation of its quality factor.

Similar concepts can be applied to other resonators, keeping in mind that a limited impact of the intra-cavity coil on the resonance mode becomes more and more difficult increasing the number of field components of the mode. In general, a single-mode resonator with high symmetry is preferable. In this respect, the proposed 'hairpin' coil can be employed in combination with a standard $\mathrm{TE}_{011}$ cylindrical cavity, in principle with similar results. It can also be applied with a rectangular cavity operating in the $\mathrm{TE}_{102}$ mode, which is relatively common in EPR. In this case, the ideal configuration would be that with flat sample holders, like those employed in the analysis of high-loss samples, in order to minimize the electromagnetic field rearrangement due to the sample holder. The application to the case of a $\mathrm{TE}_{102}$ rectangular cavity equipped with a cylindrical sample holder is expected to be still advantageous. In this case, however, a significant effect on the quality factor of the cavity is likely to be observed, due to a more complex structure for the electromagnetic fields of the resulting quasi-TE ${ }_{102}$ mode.

In the version investigated in this work, the intra-cavity rf coil can be considered as a simple and inexpensive disposable device, to the same extent as the sampleholder tube. On the other hand, a fixed mounting of the hairpin coil can be obtained by integrating it in a small tube made of a low dielectric loss material, as quartz, Teflon, of high-density polyethylene, having inner diameter compatible with the employed sample-holder tube. In this manner, the hairpin coil can be attached to the mw cavity. 


\section{Conclusions}

In conclusion, a systematic comparative study was conducted at W-band on the ENDOR performances of an intra-cavity 'hairpin' coil, with respect to external coils in the typical Helmholtz-like configuration, employing a home-made probe head based on a nr resonator. This study indicates, for the first time to our knowledge, that the higher efficiency of the intra-cavity coil is not compromised by unwanted effects as mechanical noise, thermal drift, and degradation of the mw quality factor, also at high frequency. In the broadband investigation of a $\gamma$-irradiated single crystal of LiF and a protonated BDPA in polystyrene, the gain in S/N ratio obtained with the intracavity rf coil on known ENDOR lines was about one order of magnitude at room $T$. Moreover, a complete saturation of some of these lines was observed with the hairpin coil. In the case of the BDPA in polystyrene sample, broad signals with unusual characteristics were detected, both with the intra-cavity coil and the external coils, probably originating from the sample-holder tube. The ease of construction of the hairpin coil, combined with the open structure of the $\mathrm{nr}$ resonator, suggests that the intra-cavity coil configuration investigated in this work can be profitably employed at even higher frequencies, exceeding the limit of the currently available commercial spectrometers.

Although further work is still necessary to clarify the origin of the signals with unexpected behavior, the obtained results justify a new interest in the development of very high-frequency ENDOR probe heads based on mw single-mode resonators with intra-cavity rf coil.

Acknowledgements The authors acknowledge the COST action P15 "Advanced paramagnetic resonance methods in molecular biophysics" and the Short-Term Mobility program of the National Research Council of Italy for financial support, I. Janssens for the high-level mechanical engineering of the resonator components, P. J. M. van Bentum for suggesting and providing the BDPA in polystyrene sample, and A. P. M. Kentgens for making available the rf amplifier.

Open Access This article is licensed under a Creative Commons Attribution 4.0 International License, which permits use, sharing, adaptation, distribution and reproduction in any medium or format, as long as you give appropriate credit to the original author(s) and the source, provide a link to the Creative Commons licence, and indicate if changes were made. The images or other third party material in this article are included in the article's Creative Commons licence, unless indicated otherwise in a credit line to the material. If material is not included in the article's Creative Commons licence and your intended use is not permitted by statutory regulation or exceeds the permitted use, you will need to obtain permission directly from the copyright holder. To view a copy of this licence, visit http://creativecommons.org/licen ses/by/4.0/.

\section{References}

1. G. Feher, Phys. Rev. 103, 500-501 (1956)

2. G. Feher, E.A. Gere, Phys. Rev. 103, 501-502 (1956)

3. G. Feher., Phys. Rev. 103, 834-835 (1956)

4. G. Feher, Physica 24, S80-S87 (1958)

5. M.M. Dorio, J.H. Freed, Multiple Electron Resonance Spectroscopy (Plenum Press, New York, 1979) 
6. A. Schweiger, G. Jeschke, Principles of Pulse Electron Paramagnetic Resonance (Oxford University Press, Oxford, 2001)

7. W.B. Mims, Proc. R. Soc. Lond. A 283, 452-457 (1965)

8. E.R. Davies, Phys. Lett. A 47, 1-2 (1974)

9. L. Kulik, W. Lubitz, Photosynth. Res. 102, 391-401 (2009)

10. S. Van Doorslaer, E. Vinck, Phys. Chem. Chem. Phys. 9, 4620-4638 (2007)

11. K. Moebius, A. Savitsky, High-Field EPR Spectroscopy on Proteins and Their Model Systems (The Royal Society of Chemistry, Cambridge, 2009)

12. A. Schweiger, Electron Nuclear Double Resonance of Transition Metal Complexes with Organic Ligands, in Structure and Bonding Vol. 51 (Springer, Berlin, 1982)

13. D.I. Hoult, R.E. Richards, J. Magn. Reson. 24, 71-85 (1976)

14. M. Bennati, C.T. Farrar, J.A. Bryant, S.J. Inati, V. Weis, G.J. Gerfen, P. Riggs-Gelasco, J. Stubbe, R.G. Griffin, J. Magn. Reson. 138, 232-243 (1999)

15. E.E. Motchane, J. Uebersfeld, C.R. Acad, Science 246, 1833-1835 (1958)

16. K. Gruber, J. Forrer, A. Schweiger, H.H. Gunthard, J. Phys. E Sci. Instrum. 7, 569-574 (1973)

17. J.S. Hyde, J. Chem. Phys. 43, 1806 (1965)

18. C.P. Poole, Electron Spin Resonance: a Comprehensive Treatise on Experimental Techniques (Wiley, New York, 1983)

19. J.A.J.M. Disselhorst, H. van der Meer, O.G. Poluektov, J. Schmidt, J. Magn. Reson. A 115, 183-188 (1995)

20. V. Weis, M. Bennati, M. Rosay, J.A. Bryant, R.G. Griffin, J. Magn. Reson. 140, 293-299 (1999)

21. I. Gromov, V. Krymov, P. Manikandan, D. Arieli, D. Goldfarb, J. Magn. Reson. 139, 8-17 (1999)

22. H. Blok, J.A.J.M. Disselhorst, H. van der Meer, S.B. Orlinskii, J. Schmidt, J. Magn. Reson. 173, 49-53 (2005)

23. M.M. Hertel, V.P. Denysenkov, M. Bennati, T.F. Prisner, Magn. Reson. Chem. 43, S248-S255 (2005)

24. I. Tkach, I. Bejenke, F. Hecker, A. Kehl, M. Kasanmascheff, I. Gromov, I. Prisecaru, P. Höfer, M. Hiller, M. Bennati, J. Magn. Reson. 303, 17-27 (2019)

25. O. Burghaus, M. Rohrer, T. Goetzinger, M. Plato, K. Moebius, Meas. Sci. Technol. 3, 765-774 (1992)

26. G. Annino, M. Fittipaldi, M. Martinelli, H. Moons, S. Van Doorslaer, E. Goovaerts, J. Magn. Reson. 200, 29-37 (2009)

27. G. Annino, J.A. Villanueva-Garibay, P.J.M. van Bentum, A.A.K. Klaassen, A.P.M. Kentgens, Appl. Magn. Reson. 37, 851-864 (2010)

28. J.A. Villanueva-Garibay, G. Annino, P.J.M. van Bentum, A.P.M. Kentgens, Phys. Chem. Chem. Phys. 12, 5846-5849 (2010)

29. G. Annino, in Book of Abstracts of the 4th International DNP Symposium (Elsinore, Denmark, 28-31 August 2013) p. 78; Patent WO2013000508 A1-Magnetic Resonance Hyperpolarization Probe Head

30. M. Fittipaldi, A. Cini, G. Annino, A. Vindigni, A. Caneschi, R. Sessoli, Nat. Mater. 18, 329-334 (2019)

31. G. Annino, H. Moons, S. Van Doorslaer, E. Goovaerts, in Book of Abstracts of the Joint EUROMAR 2010 and 17th ISMAR Conference (Florence, Italy, 4-9 July 2010) p. 416

32. G. Annino, H. Yashiro, M. Cassettari, M. Martinelli, Phys. Rev. B 73, 125308 (2006)

33. G. Annino, M. Cassettari, M. Martinelli, Rev. Sci. Instrum. 76, 064702 (2005)

34. G. Annino, M. Cassettari, M. Martinelli, Rev. Sci. Instrum. 76, 084702 (2005)

35. R. Tschaggelar, B. Kasumaj, M.G. Santangelo, J. Forrer, P. Leger, H. Dube, F. Diederich, J. Harmer,

R. Schuhmann, I. Garcia-Rubio, G. Jeschke, J. Magn. Reson. 200, 81-87 (2009)

36. R. Gazzinelli, R.L. Mieher, Phys. Rev. 175, 395-411 (1968)

37. Y.H. Chu, R.L. Mieher, Phys. Rev. 188, 1311-1319 (1969)

38. M.L. Dakss, R.L. Mieher, Phys. Rev. Lett. 18, 1056-1058 (1967)

39. M.L. Dakss, R.L. Mieher, Phys. Rev. 187, 1053-1061 (1969)

40. B. Epel, D. Arieli, D. Baute, D. Goldfarb, J. Magn. Reson. 164, 78-83 (2003)

41. G. Annino, M. Cassettari, M. Martinelli, IEEE Trans. Microwave Theory Tech. 57, 775-783 (2009)

Publisher's Note Springer Nature remains neutral with regard to jurisdictional claims in published maps and institutional affiliations. 